\author{
Military Technical College \\ Kobry El-Kobbah, \\ Cairo, Egypt.
}

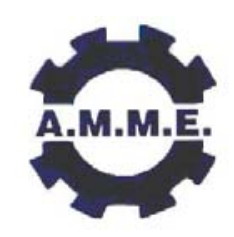

$13^{\text {th }}$ International Conference on Applied Mechanics and Mechanical Engineering.

\title{
BUCKLING ANALYSIS OF CARBON NANOTUBES USING A BEAM ELEMENT BASED ON MOLECULAR MECHANICS
}

\author{
$\mathrm{HU}^{*} \mathrm{~N} ., \mathrm{NONUYA}^{* *} \mathrm{~K}$. and FUKUNAGA ${ }^{* *} \mathrm{H}$.
}

\begin{abstract}
Based on both molecular mechanics and computational structural mechanics, a threedimensional (3D) equivalent beam element is developed to model a $\mathrm{C}-\mathrm{C}$ covalent bond on carbon nanotubes (CNTs) whereas the van der Waals forces between atoms in the different walls of multi-walled CNTs are described using a rod element. The buckling characteristics of CNTs are conveniently analyzed by using the traditional finite element method (FEM) of a 3D beam and rod model, termed as molecular structural mechanics approach (MSMA). Moreover, to model the CNTs with large length or large diameter, the validity of Euler's beam buckling theory and a shell model with proper properties defined from the results of MSMA is investigated. The predicted results by this simple continuum mechanics approach agree well with the reported experimental data.
\end{abstract}

\section{KEY WORDS}

Carbon nanotube, Buckling analysis, Molecular mechanics, Finite element analysis

\footnotetext{
${ }^{*}$ Associate professor, Dpt. of Aerospace Engineering, Tohoku University, Sendai, Japan. ${ }^{* *}$ Graduate student, Dpt. of Aerospace Engineering, Tohoku University, Sendai, Japan. ${ }^{* * *}$ Professor, Aerospace Engineering, Tohoku University, Sendai, Japan.
} 


\section{INTRODUCTION}

The unique geometry (small diameter and high aspect ratio) and exceptional mechanical properties (high stiffness, high strength and resilience) of CNTs make them ideal for applications such as probe tips of scanning probe microscope (SPM) and tips of nano-indenter [1-3]. Thus, it is imperative to understand the buckling behaviors of CNTs under axial compression for those applications. A fundamental challenge to achieve this, however, is to develop a proper mechanistic model at nanoscale. Although there have been some studies in this field [3-6], to the best of authors' knowledge, there have been almost no reliable experimental verifications for the published analytical results. In this paper, based on both molecular mechanics and computational structural mechanics, we propose a novel numerical model termed as molecular structural mechanics approach (MSMA), to study the buckling characteristics of CNTs. Additionally, based on the Euler's beam buckling theory and Timoshenko's shell buckling theory, a simple continuum mechanics approach is established with experimental validations. An important finding from the results is that some representative properties of CNTs in the buckling analysis of the above continuum mechanics model are size dependent.

\section{THEORY}

Based on the second-generation molecular-force field and computational structural mechanics, we have proposed a 3D structural beam element to model the covalent C-C bond, as shown in Fig. 1 [7]. The stiffness parameters of this equivalent beam element, e.g. extensional stiffness $E A_{B}$, bending rigidity $E I_{B}$ and torsion rigidity $G J_{B}$, are expressed as follows [7]:

$$
\begin{aligned}
& E A_{B}=K_{s} R_{0} \\
& E I_{B}=\frac{K_{s} R_{0}^{3}\left(K_{s} R_{0}^{2}+3 K_{\theta}\right)}{36\left(K_{s} R_{0}^{2}-K_{\theta}\right)} \\
& G J_{B}=2 V_{\omega} R_{0}
\end{aligned}
$$

where $R_{0}$ is the equilibrium bond distance, which is $0.142 \mathrm{~nm}$ for C-C bonds, $K_{s}$ the force constant of bond stretching, $K_{\theta}$ the angle bending force constant and $V_{\omega}$ is the torsional barrier. As shown in [7], we take $K_{s}=805.5 \mathrm{nN} / \mathrm{nm}, K_{\theta}=1.438 \mathrm{nN} \cdot \mathrm{nm} / \mathrm{rad}^{2}$ and $V_{\omega}=0.101 \mathrm{nN} . \mathrm{nm}$. This results in the following Young's modulus and Poisson's ratio of CNTs: $E=1.06 \mathrm{TPa}$ and $v=0.225[7]$.

A finite element (FE) beam model consisting of the above beam elements is used in this study for the buckling analysis of single-walled CNTs. For the multi-walled CNTs, besides the above beam element, a rod element has been newly developed here as follows to account for the van der Waals forces between $\mathrm{C}$ atoms on the different walls. As shown in Fig. 2, for one carbon atom on the inner wall, there are some rod elements, which connect it with some carbon atoms on the outer wall. The stiffness of the rod element will be modeled with a Lennard-Jones potential with the cutoff distance of $1.2 \mathrm{~nm}$. Here, Lennard-Jones 6-12 potential is used to describe the interactions of atoms locating on two neighboring walls 

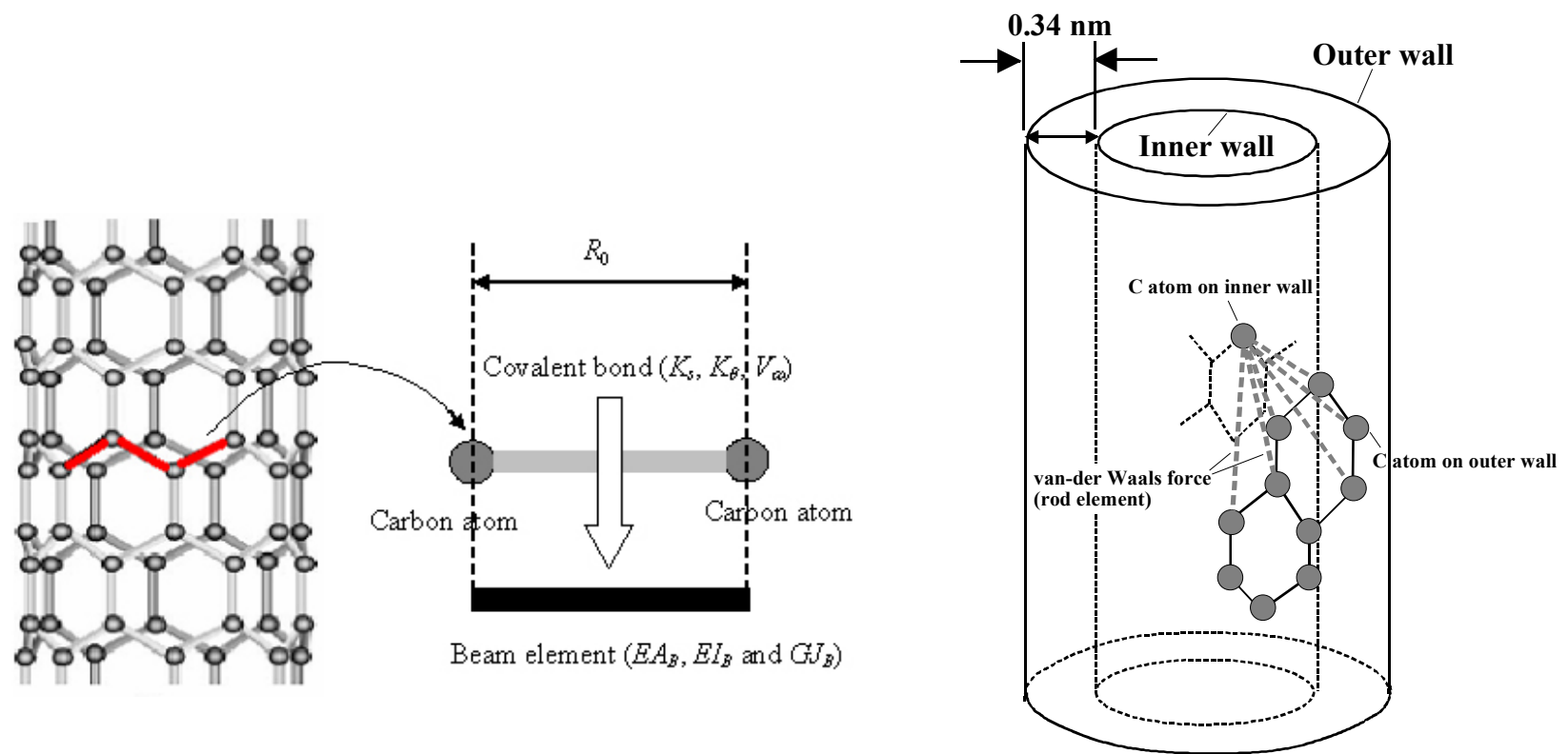

Fig.1 Schematic view of construction of beam element for $\mathrm{C}-\mathrm{C}$ bond

$U(R)=4 \varepsilon\left[\left(\frac{\sigma}{R}\right)^{12}-\left(\frac{\sigma}{R}\right)^{6}\right]$

where $R$ is the interatomic distance, and the Lennard-J ones parameters, $\varepsilon$ and $\sigma$, are $3.8655 \times 10^{-4} \mathrm{nN} . \mathrm{nm}$ and $0.34 \mathrm{~nm}[5]$, respectively.

Taking $R_{1}$ as the initial distance between two carbon atoms located on two different walls, which is usually set to be $0.34 \mathrm{~nm}$, due to the infinite small change of distance by $\Delta R$, by neglecting the terms beyond the 3rd order, the variation of potential can be obtained as

$$
\Delta U \approx \frac{d U\left(R_{1}\right)}{d R} \Delta R+\frac{d^{2} U\left(R_{1}\right)}{2 d R^{2}} \Delta R^{2}
$$

For a rod element, the change of strain energy can be described by

$$
\Delta \Gamma_{R}=F \Delta R+\frac{E A_{R}}{2 R_{1}} \Delta R^{2}
$$

By comparing Eqs. (5) and (6), we can obtain the extensional stiffness of rod element as

$$
E A_{R}=R_{1} \frac{d^{2} U\left(R_{1}\right)}{d R^{2}}
$$

The initial van der Waals force at the equilibrium state can be evaluated as

$$
F=\frac{d U\left(R_{1}\right)}{d R}
$$


Thus, the final equation for the FEM buckling analysis can be written as

$\left[\boldsymbol{K}_{S}+\boldsymbol{K}_{V S}\right]\{\boldsymbol{u}\}=\lambda_{\min }\left[\boldsymbol{K}_{G}+\boldsymbol{K}_{V G}+\boldsymbol{K}_{G 0}\right]\{\boldsymbol{u}\}$

where $\boldsymbol{K}_{s}$ is the stiffness matrix of beam elements, and $\boldsymbol{K}_{v s}$ is the stiffness matrix of rod elements, $\boldsymbol{K}_{G}$ is the geometric stiffness matrix of beam elements, $\boldsymbol{K}_{V G}$ is the geometric stiffness matrix of rod elements, and $\boldsymbol{K}_{G 0}$ is the initial geometric stiffness matrix of rod elements due to the initial van der Waals forces. Finally, the final buckling load is determined as $F_{c r}=\lambda_{\min }$ for $\left(\lambda_{\min }>0\right)$. Here, an efficient 3 -noded strain beam element proposed by authors [8] is adopted, and the rod element is modeled by using the same beam element with very small bending stiffness.

\section{VERIFICATION AND INVESTIGATION}

To validate our MSMA for single- and double-walled CNTs with the fixed-fixed boundary condition, the critical compressive strain obtained by MSMA has been compared with that obtained by classical MD computations [4]. In Fig. 3, the present results exhibit excellent agreement with the results by MD computations in [4]. Also, it is found that the buckling of CNTs can be divided into two categories. The one is the shell buckling mode when the length of CNT is very small or the aspect ratio is very low. With the increase of aspect ratio of CNT, the buckling mode was observed to change into a beam or Euler buckling mode.

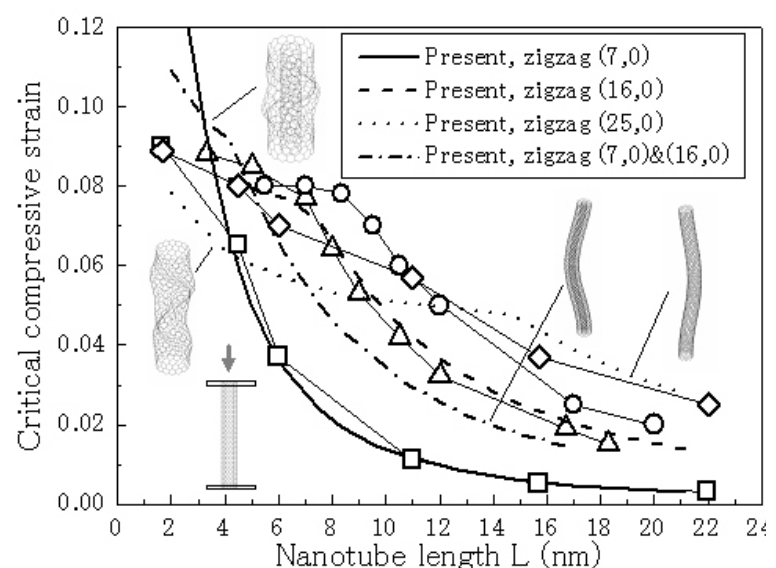

Fig. 3 Comparison of buckling load,-口-MD, zigzag $(7,0)[4]$; -O- MD, zigzag $(16,0)[4]$; $\checkmark-M D$, zigzag $(25,0)[4] ; \triangleleft-M D$, zigzag $(7,0) \&(16,0)[4]$

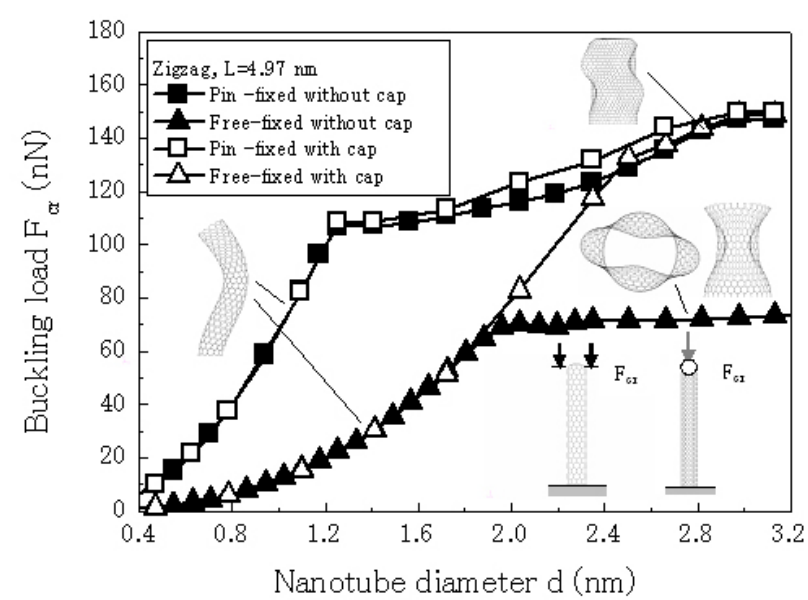

Fig. 4 Buckling loads of single-walled CNT

With the validation of the MSMA, we calculated the buckling loads of single-walled CNTs with and without end caps as shown in Fig. 4, under the pin-fixed and free-fixed boundary conditions. From the results, it is found that for Euler buckling, both kinds of results are identical. However, for shell buckling, the buckling loads of CNT with cap are around $50 \%$ higher than those of CNT without cap. Therefore, for the case of shell buckling mode, the consideration of effect of cap on the buckling load is crucial. Also, for shell buckling, the buckling behavior of the capped CNTs with the free-fixed boundary condition is equivalent to those of the capped or uncapped CNTs with the pin- 
fixed boundary condition. For multi-walled CNTs, we got the following conclusion: The buckling loads of multi-walled CNTs in the shell buckling mode can be simply obtained from the product of the wall number and the buckling loads of single-walled CNTs.

As a CNT typically is composed of millions of carbon atoms, further simplification is needed. First, we deal with CNTs with high aspect ratios, where the Euler buckling theory can be applied,

$$
F_{c r}=\frac{\pi^{2} E I}{(k L)^{2}}
$$

where $k$ is a constant determined by boundary conditions, $E I$ and $L$ are the bending rigidity and the length of a CNT.

First, we investigate the effectiveness of Euler's beam buckling theory by comparing with the present MSMA results as shown in Fig. 5 . The results are normalized by the moment of inertia of the cross-sectional area of the beam. For multi-walled CNTs, the total thickness of several walls is used for calculating the moment of inertia. From this figure, it can be found that the results of Euler's bema buckling theory agree with those of MSMA very well. For multi-walled CNTs, we can get the conclusion that the increase of wall number is equivalent to increase of the moment of inertia of the cross-section in the case of Euler buckling.

To further verify the effectiveness of Euler's beam buckling theory, the experimental results [3] for CNTs with high aspect ratios are used in this study. For the experimental setup [3], at the top of CNTs, the boundary condition can be considered to be a fixed one. At the bottom of CNTs, the boundary condition can be considered to be a pin connection. Under this boundary condition, in Eq. (10), $k$ is equal to 0.7 . However, this pin connection is usually not perfect, and then, $k=0.8$ in [9], is also used. For specimens $A$ and $B$ in Table 1, the calculated results with the experimental ones are listed in Table 2 , which shows the effectiveness of the Euler buckling theory. One can find that the calculated buckling loads based on the recommended value, $k=0.8$, are more close to the experimental ones than those of $k=0.7$.

Second, Waters et al. [6] performed the shell buckling experiments of 15-walled CNTs with the outer diameter of $50.0 \mathrm{~nm}$ and the inner diameter of $40.0 \mathrm{~nm}$. The lengths of CNTs are $50.0 \mathrm{~nm}$ and $100.0 \mathrm{~nm}$, respectively. First, a single-wall (length: $50.0 \mathrm{~nm}$, diameter: $45.0 \mathrm{~nm}$ ) in this 15-walled CNT [6] was analyzed by FEM using a shell element in ANSYS ${ }^{\top M}$. The properties of this shell are adjusted to make its results match the ones of present MSMA, which leads to: $E=920 \mathrm{GPa}, \quad v=0.225$, and thickness $h=0.218 \mathrm{~nm}$. However, for Euler beam buckling, we use: $E=1.06 \mathrm{TPa}$ and thickness $h=0.34 \mathrm{~nm}$. This difference emanates from the different deformation behaviors in two buckling modes, which shows that some important properties of CNTs are size dependent or deformation dependent in a strict sense. The Timoshinko's shell buckling theory is also employed as:

$$
P_{c r}=\frac{2 \pi E h^{2}}{\sqrt{3\left(1-v^{2}\right)}}
$$




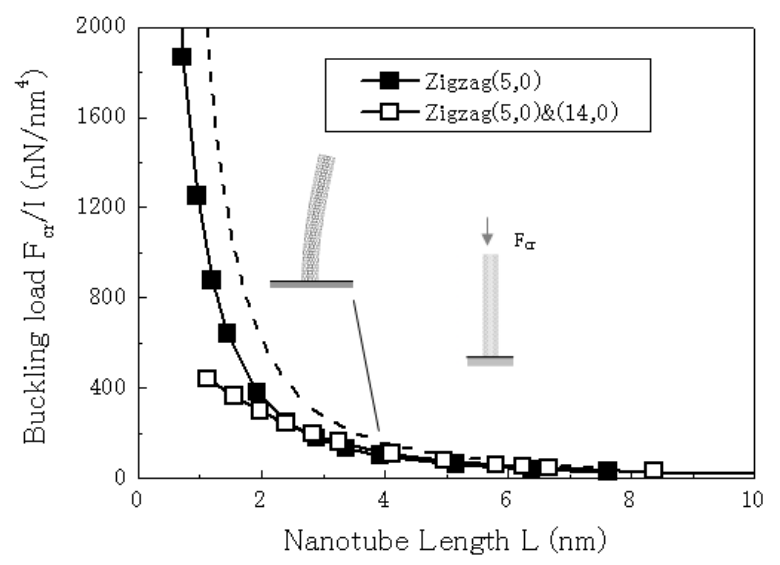

Fig. 5 Buckling loads of MSMA and Euler's beam buckling theory for single-walled and two-walled CNTs - - - Euler's result

Table 2. Comparison of results of Euler's theory and experiments [3]

\begin{tabular}{|c|c|c|c|}
\hline & $k=0.7$ & $k=0.8$ & Experimental [3] \\
\hline (A) & $33.9 \mathrm{nN}$ & $26.5 \mathrm{nN}$ & $24.5 \mathrm{nN}$ \\
\hline (B) & $31.8 \mathrm{nN}$ & $24.8 \mathrm{nN}$ & $24.0 \mathrm{nN}$ \\
\hline
\end{tabular}

Table 1. Parameters of carbon nanotube specimens [3]

\begin{tabular}{|l|c|c|}
\hline & $(\mathrm{A})$ & $(\mathrm{B})$ \\
\hline Length (um) & 1.19 & 1.07 \\
\hline Outer diameter (nm) & 14.7 & 14.7 \\
\hline Inner diameter (nm) & 1.3 & 10.3 \\
\hline Number of walls & 20 & 6 \\
\hline
\end{tabular}

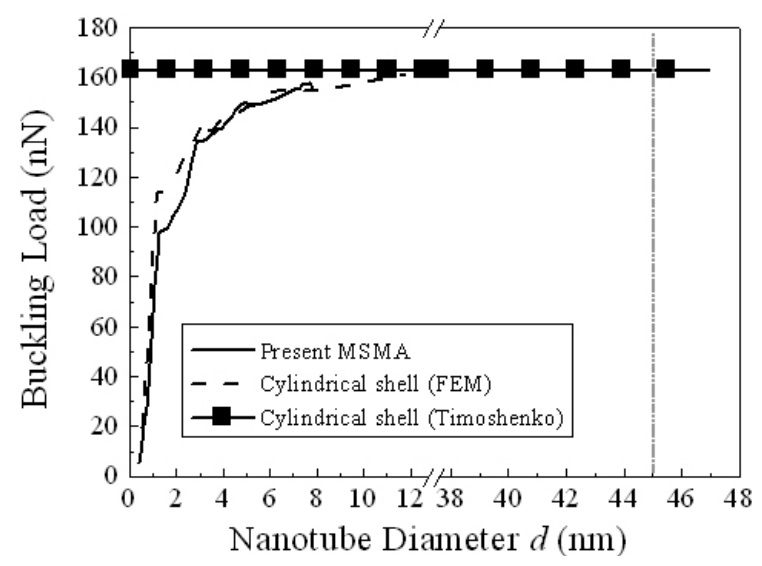

Fig. 6 Comparison of results of MSMA, FEM shell model and Timoshenko's theory

We note that the above material properties of the stretch stiffness of a graphite sheet is $201 \mathrm{~N} / \mathrm{m}$ calculated from $\mathrm{E} h$. This value is approximately $40 \%$ lower than that obtained by atomistic calculations, which is around $360 \mathrm{~N} / \mathrm{m}$. One way to avoid this discrepancy is to employ the Young's modulus and thickness of nanotube as $2.964 \mathrm{TPa}$ and 0.1213 $\mathrm{nm}$, respectively. In this case, the stretch stiffness of the graphite sheet matches the previous result of atomistic calculations. Moreover, we have checked the buckling load of FEM shell model using these material properties, which yields the almost same result with that by the above material properties. 1he reason is that the shell buckling load is dominated by $E h^{2}$ which is identical for both kinds of material properties.

A comparison of buckling loads by three approaches is shown in Fig. 6, revealing that in the stage of shell buckling all three methods yield the same result and the buckling loads are virtually independent on the length and diameter of CNT. For a single wall in the 15-walled CNT [6], the buckling load predicted by the Timoshinko's shell buckling theory of Eq. (11) is $162.7 \mathrm{nN}$. After timed by 15, i.e. the number of walls, the buckling load of the CNTs used by Waters et al. [6] is calculated to be $2.44 \mu \mathrm{N}$, which agrees well with the reported experimental results, i.e., between $2.0 \mu \mathrm{N}$ and $2.5 \mu \mathrm{N}$.

\section{SUMMARY}

We have developed a numerical model based on molecular mechanics and computational structural mechanics, i.e., MSMA. The buckling characteristics of CNTs 
are analyzed by MSMA. Also, a continuum mechanics approach based on the Euler's beam buckling theory and a shell model is studied. The obtained results by this simple model agree well with the reported experimental data.

\section{REFERENCES}

[1] Meyyappan, M., Carbon Nanotubes: Science and Applications, CRC press, LLC (2004).

[2] Dai, H., Hafner, J.H., Rinzler, A.G., Colbert, D.T. and Smally, R.E., "Nanotubes as nanoprobes in scanning probe microscopy", Nature, No. 384, pp. 147-150, (1996).

[3] Akita, S., Nishijima, H., Kishida, T. and Nakayama, Y., "Nanoindentation of polycarbonate using carbon nanotube tip", Jpn. J. Appl. Phys., No. 39, pp. 70867089, (2000) (in Japanese).

[4] Sears, A. and Batra, R.C., "Macroscopic properties of carbon nanotubes from molecular-mechanics simulations", Phys. Rev. B, No. 69, pp. 235406 , (2004).

[5] Li, C. and Chou, T.W., "A structural mechanics approach for the analysis of carbon nanotubes" Int. J. Solids and Struct., No. 40, pp. 2487-2499, (2003).

[6] Waters, J. F., Guduru, P. R., Jouzi, M. and Xu, J. M., "Buckling of individual multiwalled carbon nanotubes using nanoindentation", Appl. Phys. Lett., No. 87, pp. 103109, (2005).

[7] Hu, N., Fukunaga, H., Lu, C., Kameyama, M. and Yan, B., "Prediction of elastic properties of carbon nanotube-reinforced composites", Proc. Royal Soc. London. Series A. Math. and Phys. Sci., No. 461, pp. 1685-1710, (2005).

[8] Hu, N., Hu, B., Yan, B., Fukunaga, H. and Sekine, H., "Two kinds of $\mathrm{C}^{0}$-type element for buckling analysis of thin-walled curved beam", Comp. Meth. Appl. Mech. and Eng., No. 171, pp. 87-108, (1999).

[9] SSRC (Structure Stability Research Council), Guide to Stability Design Criteria for Metal Structures, John, Wiley \& Sons (1976). 